Revista Tecnologia e Sociedade, Curitiba, v. 11, n. 23, 2015

ISSN (versão online): 1984-3526

ISSN (versão impressa): 1809-0044

\title{
Sistemas inteligentes? Humanos dependentes?
}

Intelligent systems? Dependent human beings?

Waldemar Bonventi Jr. ${ }^{1}$

Artigo submetido em out./2014 e aceito para publicação em abr./2015.

\section{RESUMO}

Discute-se o impacto das tecnologias de informação e comunicação, estendendo-se aos dispositivos e sistemas industriais mais complexos. São apresentados os sistemas inteligentes e seu contraponto mercadológico: sistemas divulgados como inteligentes. Textos acadêmicos sobre inteligência artificial são referenciados como base conceitual para a análise dos impactos sociais das tecnologias inteligentes ou assim divulgadas. Questões sobre raciocínio e busca de soluções, utilizando o jogo de xadrez como comparação entre seres humanos e computadores, procuram lançar luz sobre os processos mentais que são relacionados com comportamento inteligente. Questiona-se a dependência ou confiança das pessoas em deixarem decisões mais complexas para tais dispositivos ou sistemas, desde simples cálculos aritméticos até inferências e proposta de ações estratégicas, passando pela interação entre sistemas inteligentes e seres humanos para melhorar a eficiência de tarefas complexas em geral. Por fim, são postas questões reflexivas sobre a real possibilidade da inteligência artificial suplantar a humana, criando involuntariamente uma realidade virtual e como atualmente a tecnologia possivelmente está criando esta realidade e uma nova forma de dependência.

Palavras-chave: Sistemas inteligentes. Cibernética. Inteligência artificial. Sistemas automáticos. Impactos sociais da automação.

\begin{abstract}
We discuss the impact of information and communication technologies, extending to more complex devices and industrial systems. Intelligent systems and its market counterpoint disclosed as intelligent systems, are presented. Academic texts on artificial intelligence are referenced as a conceptual basis for sustaining the social impacts of smart technologies expansion or disclosed as well. Questions about thinking and searching for solutions using the game of chess as a comparison between humans and computers, seeking to shed light on the mental processes that are related to intelligent behavior. We question the reliance or trust of people in more complex decisions to leave such devices or systems, from simple arithmetic to inferences and propose strategic actions, through the interaction between humans and intelligent systems to improve the efficiency of tasks complex as a whole. Finally, reflective questions are posed about the real possibility of artificial intelligence supplant humans, creating a virtual reality and how technology can currently be creating this reality and a new form of dependency.
\end{abstract}

Keywords: Intelligent systems. Cybernetics. Artificial intelligence. Automated systems. Social impact of automation.

\section{INTRODUÇÃO}

Frequentemente lemos ou ouvimos a expressão "sistemas inteligentes". Qual

\footnotetext{
${ }^{1}$ Doutor em Engenharia de Computação. Docente do Programa de Mestrado Profissional em Processos Tecnológicos e Ambientais. Universidade de Sorocaba. E-mail: waldemar.bonventi@prof.uniso.br.
} 
Revista Tecnologia e Sociedade, Curitiba, v. 11, n. 23, 2015

ISSN (versão online): 1984-3526

ISSN (versão impressa): 1809-0044

seu significado no contexto tecnológico atual? O que caracteriza tais sistemas? Que objetos, utensílios, produtos, processos ou mesmo sistemas podem ser classificados nessa categoria e como - para fins de consumo - têm sido "anunciados"? Atualmente, o avanço tecnológico está tornando tênue a separação entre sistemas automáticos controlados por uma extensa ou complexa programação dos seus componentes e os sistemas a que se pode atribuir comportamento inteligente através de métodos desenvolvidos pela Inteligência Artificial.

Este artigo segue a introduzir uma reflexão em dois assuntos: as tecnologias empregadas em aparelhos utilizados diretamente (ou quase) pelas pessoas possuem inteligência de fato e suficiente para auxiliar ou substituir decisões humanas de certa complexidade? A dependência humana desta categoria de aparelhos pode afetar a maneira de interagir com o mundo externo, levando a uma virtualização ou distorção excessiva da realidade percebida?

São reunidas aqui várias informações extraídas de revistas, noticiários, artigos, selecionados de maneira amostral como referenciais às questões de comportamento inteligente, automação, suas conceituações e mesmo a inteligência existente no comportamento humano. Este texto faz uso de um método qualitativo de estudo, a fim de promover uma reflexão sobre a escalada dos sistemas inteligentes e sua inserção na sociedade. Qualquer maneira de quantificação do impacto resultante é hoje imprecisa e limitar-se-ia às estatísticas de vendas e de utilização, coletadas de uma lista de aparelhos comuns no meio social.

\section{O USO COTIDIANO DA TECNOLOGIA}

Cada vez mais somos surpreendidos com a evolução da tecnologia e o aumento da capacidade e das funções dos utensílios cotidianos. Em nossos smartphones, temos inúmeras funções que os aproximam de computadores completos.

Há muitos modos de se enviar mensagens para quem não possa atender àquela chamada realizada em um algum momento, via SMS, e-mail, Facebook Messenger, Whatsapp, Hangouts e outros. Se as teclas do aparelho forem pequenas, sensíveis ou difíceis de visualizar, pode-se ditar a mensagem. Os sons das palavras são reconhecidos transformados em texto a ser enviado. Ainda é necessário inserir os pontos e as vírgulas, a fim de manter a ortografia. Mas a 
Revista Tecnologia e Sociedade, Curitiba, v. 11, n. 23, 2015

ISSN (versão online): 1984-3526

ISSN (versão impressa): 1809-0044

maioria das pessoas não têm se preocupado muito com isso.

Além disso, outros dispositivos podem ser analisados sob esta ótica. $\mathrm{O}$ mercado oferece muitos dispositivos ou sistemas denominados "inteligentes". No filme "Eu, Robô" (2004, baseado no livro de Isaac Asimov), existem androides prestadores de serviços tal qual Rosie, a doméstica robótica da Família Jetson (PEREIRA, 2006; NOVAK, 2012). Recentemente foram lançados aspiradores de pó que percorrem todo o ambiente desviando-se de obstáculos e penetrando sob a mobília. Mais: se a bateria estiver no final, retornam à base, se recarregam e voltam ao ponto onde estavam (ULRICH; MONDADA; NICOUD, 1997).

Uma janela inteligente é capaz de controlar a luminosidade de ambientes, como um importante recurso para economizar energia elétrica. Pode mudar de cor ao receber impulsos elétricos. É constituída de um "sanduíche" de diferentes materiais "inteligentes" cuja constituição química lhes permite responderem a um determinado estímulo. E pode se transformar em uma tela sensível ao toque ou num LCD transparente, para ler os e-mails, consultar a internet, gerar uma imagem ... como um "tablet" gigante (LAMPERT, 1998). Teremos roupas inteligentes, que poderão ler a nossa frequência cardíaca e respiratória e capazes de libertar materiais ativos à pele, como antitranspirantes, antibacterianos ou hidratantes (AXISA et al, 2005). Semáforos inteligentes podem controlar o fluxo de veículos em cruzamentos, em função do horário, da via mais ocupada e até de contingências, como acidentes, fiscalizações e outros. (MESE et al, 2006).

Pesquisa-se materiais equipados que "se comportam" como sistemas biológicos. Estes "materiais inteligentes" consertam-se por conta própria ou se adaptam a certas condições do ambiente. Necessitam de atuadores e motores que desempenho o mesmo papel dos músculos, nervos e memória. Um computador ligado a eles faz a vez do cérebro. Tubos metálicos (stents) estão sendo inseridos em artérias para evitar seu entupimento. Em breve, tomarão a forma necessária ao atingirem a temperatura corporal (IIDA et al, 2013). "Materiais com memória" depois de deformados retornam à sua forma original, estimulados por calor ou luz (GALL et al, 2005). Piezocerâmicas convertem vibração em energia elétrica e também vibram ao recebê-la. Desenvolvem-se "esferas inteligentes" que serão empregadas em rolamentos de veículos, controladas para neutralizar as vibrações durante o movimento. O mecanismo ativo anula a vibração tão logo ela seja detectada. Ainda, o material piezelétrico pode gerar energia elétrica e alimentar as baterias de um 
Revista Tecnologia e Sociedade, Curitiba, v. 11, n. 23, 2015

ISSN (versão online): 1984-3526

ISSN (versão impressa): 1809-0044

veículo (BOZCHALUI; JIN; SHARMA, 2014).

Polímeros são testados para revestir tanques de combustível. Havendo uma perfuração ou rasgo, as moléculas se rearranjam para "cicatrizar o ferimento" (SNIHIROVA et al, 2014). O robô Asimo da Honda caminha e gesticula como um ser humano. Mostra capacidade de compreensão dos gestos das pessoas e responde a perguntas com raciocínio próprio (CAO et al, 2013). Uma casa inteligente acende e apaga as luzes via sensores que medem a quantidade de luz, gerando uma grande economia para seus moradores. Se entrar muito sol, a casa fecha as cortinas para diminuir o uso do ar-condicionado. As luzes são acesas só quando há alguém dentro da casa (RASHID et al, 2013).

\section{PROPAGANDA COMERCIAL}

Os sistemas e dispositivos citados são inteligentes? Ou serão apenas produto de automação pura e simples? Em um sistema automático, os mecanismos efetuam medições e fazem correções, sem a interferência do homem. Contêm sensores que captam os dados do ambiente (sons, imagens, tensões, pressões, correntes elétricas, temperaturas, etc.), enviam ao módulo que contém a programação do "que fazer" com estes dados e controlam um conjunto de "atuadores", que interagem com o mesmo ambiente para controlá-lo (OGATA, 2011). No aparelho de ar condicionado, se a temperatura sobe, ele esfria o ambiente. Logo, seus sensores "avisam" para diminuir o ritmo de resfriamento e manter a temperatura nos limites predefinidos. Em um dispositivo mais complexo, como os existentes em veículos repletos de computação embarcada, a programação de controle embutida em seus microprocessadores contém milhões de palavras em linguagens de programação.

Então, o que está no programa de controle são regras definidas pelos engenheiros. Estas seguem um modelo matemático rígido. Regras lógicas definem o "comportamento" do controlador. Ou seja, a lógica vem do raciocínio do programador, que escreveu as regras no programa.

Estes sistemas automáticos aprendem? Planejam? Compreendem ideias? Criam soluções? As janelas, roupas, semáforos, materiais sintéticos, stents, esferas de rolamentos, polímeros dos tanques, residências e tantos outros equipamentos e objetos são capazes disso?

Um documento oficial elaborado para o governo brasileiro define "produto 
Revista Tecnologia e Sociedade, Curitiba, v. 11, n. 23, 2015

ISSN (versão online): 1984-3526

ISSN (versão impressa): 1809-0044

inteligente" como

"produto dotado de uma capacidade de processamento eletrônico, que adequa seu comportamento em função de sensoriamento, comandos e/ou dados fornecidos, atuando e desempenhando com alta versatilidade e eficiência suas finalidades. Exemplos de produtos inteligentes são: Impressora de jato de tinta, airbag, ABS, TV digital, reatores químicos, telefones móveis, cabeças magnéticas de discos de armazenamento, eletrodomésticos em geral, sensores e atuadores biomédicos para implante, analisadores químicos, analisadores de DNA, smart-cards, monitores de processos industriais, e muitos outros" (CAPDA, 2004).

Estes não seriam apenas resultantes de automação pura (talvez não tão simples)? O que aconteceria quando ocorresse uma resposta ou comportamento "errados"? O sistema teria algum processo de avaliação e correção de erros? Poderia "aprender com seus erros"?

Em 1993, um grupo de trabalho foi criado pelo Comitê Técnico de Controle Inteligente do IEEE Control Systems Society para definir as características do Controle Inteligente, evitando a questão da inteligência em geral. A ênfase foi em definições úteis, não em nomenclaturas. Concluiu-se que "um sistema inteligente deve ser altamente adaptável para significativas mudanças inesperadas, e assim a aprendizagem é essencial. E deve exibir um alto grau de autonomia no tratamento das mudanças." (CASTRO; CARVALHO; SANTOS, 2014). Então, quais sistemas ou dispositivos podem receber o adjetivo "inteligente"?

\section{COMBINAÇÃO HOMEM-MÁQUINA}

Locais inacessíveis (fundo do mar, fora do planeta, usinas nucleares) ou insalubres (agentes nocivos ou tóxicos), tarefas repetitivas em que falhas podem comprometer a segurança ou a produtividade, análise de enormes quantidades de dados, em todos são aplicáveis sistemas autônomos que apresentem comportamento inteligente. Áreas como "instrumentação inteligente" e "automação inteligente" têm se desenvolvido para aperfeiçoar a combinação homem-máquina. (REZENDE, 2003)

Um site na internet sobre tecnologia noticia que um smartphone funciona como controle remoto de vários aparelhos eletrônicos integrados em um sistema de automação residencial e contém e disponibiliza remotamente dados pessoais. Este site é aberto a comentários de usuários cadastrados. Um deles para reflexão: "Casa inteligente, morador burro, pré-programado. Hoje é sua empregada monitorada, amanhã vai ser você no seu trabalho. Fora que o ladrão pega seu celular e sua vida 
Revista Tecnologia e Sociedade, Curitiba, v. 11, n. 23, 2015

ISSN (versão online): 1984-3526

ISSN (versão impressa): 1809-0044

está na mão dele..." Aí também entra a alternativa da identificação biométrica: são dezenas de características físicas individuais únicas (DELAC; GRGIC, 2004) para evitar este tipo de problema. Que traz outros: no filme Minority Report (BOSCOV, 2002), o detetive John Anderton (Tom Cruise) precisa substituir sua identificação feita pela íris ocular. Baseado em um conto de Philip K. Dick (2002), o filme associa a campanha da Presidência americana para limitar as liberdades civis em troca de maior agilidade para prender suspeitos de terrorismo. E isto antes do 11 de Setembro!

Há uma ou duas gerações, as aulas de Matemática para o Ensino Fundamental eram ministradas por professores com sólida base, mesmo formados pedagogos. Álgebra, logaritmos e radiciação manual eram conteúdo obrigatório. No Ensino Superior, como não havia "mineração de dados", os modelos matemáticos da realidade eram duramente construídos com base dados empíricos e o conhecimento evoluía via testes exaustivos desses modelos. Engenheiros faziam extensos memoriais de cálculo do projeto, antes de executá-lo.

A década de 80 trouxe a popularização do computador, e a de 90 , a da Internet. Processamento de propósito geral e comunicação "ponto-a-ponto" alastraram-se do nível corporativo para o individual. Interfaces homem-máquina amigáveis possibilitaram o aprendizado amplo da operação destes dispositivos por crianças. Sistemas multimídia favoreceram a interdisciplinaridade entre as áreas educacionais e tecnológicas.

Então, a calculadora eletrônica, o computador, o software de modelagem e de simulação permitiram que os humanos ganhassem tempo. Projetos complexos nascem rapidamente e rapidamente são modificados. A indústria pode renovar (e inovar) seus produtos com uma frequência assustadora.

Dispomos de ferramentas mais poderosas para a tarefa "pesada" ou "enfadonha". Não se perde tempo com cálculos aritméticos simples. Conversão de unidades, tais como de quilômetros para metros, são feitas por alunos com calculadoras, não com o cérebro (e muitas vezes perdem tempo para revirar a mochila e tirá-las).

\section{INTELIGÊNCIA E SISTEMAS INTELIGENTES}

Mas o que é inteligência? Pode ser definida como a capacidade mental de 
Revista Tecnologia e Sociedade, Curitiba, v. 11, n. 23, 2015

ISSN (versão online): 1984-3526

ISSN (versão impressa): 1809-0044

raciocinar, planejar, resolver problemas, abstrair e compreender ideias, linguagens e aprender. Na Psicologia, entende-se que este conceito não compreende a criatividade, o caráter ou a sabedoria. Conforme a definição que se tome, pode ser considerado um dos aspectos da personalidade (GOTTFREDSON, 1997). As pessoas têm insights, ou como muitos dizem, "acendeu-se uma luz" ao surgir uma ideia ou solução nova.

Mas, e a criatividade? Não faria parte disso? (JAUK et al, 2013) O mais interessante é que, ao pedir para alguém explicar como surgiu essa ideia ou solução, dificilmente será em termos de uma sequência de raciocínio ou encadeamento de outras ideias. Muitos afirmam que a ideia veio "espontaneamente". Muito se credita ao "subconsciente", que fica trabalhando em segundo plano na solução, sem "atrapalhar" nosso raciocínio imediato ou nossos afazeres.

O psicólogo Howard Gardner desenvolveu a teoria das inteligências múltiplas, identificando sete diferentes tipos: lógico-matemática, linguística, espacial, musical, cinemática, intrapessoal e interpessoal, expandindo posteriormente aos conceitos de inteligência naturalista e a existencial (GARDNER, 1999). Esta discussão ficaria extensa aqui, desde o "teste de Ql" até vários outros, a fim de "medir" a inteligência.

Voltando ao tema principal - sistemas inteligentes - pergunta-se: o que é inteligência artificial? É uma área de pesquisa da computação dedicada a buscar métodos computacionais que possuam ou multipliquem a capacidade racional do ser humano de resolver problemas, pensar ou, de forma ampla, ser inteligente. Também é definida como "o ramo da ciência da computação que se ocupa do comportamento inteligente" ou ainda, "o estudo de como fazer os computadores realizarem coisas que, atualmente, os humanos fazem melhor". O principal objetivo destes sistemas é executar funções que, caso um ser humano as executasse, seriam consideradas inteligentes. É um conceito amplo que recebe tantas definições quanto se dá significados diferentes à inteligência (RUSSELL; NORVIG, 2013; COPPIN, 2010).

É aí que começam os problemas. Para ilustrar, Deep Blue foi um supercomputador criado pela IBM especialmente para jogar xadrez, utilizando 256 processadores. Seu programa era capaz de analisar aproximadamente 200 milhões de posições por segundo (CAMPBELL; HOANE; HSU, 2002). Em fevereiro de 1996, o campeão mundial de xadrez, Gary Kasparov, considerado o melhor de todos os tempos, ganhou três partidas, empatou duas e perdeu uma para Deep Blue. A única 
Revista Tecnologia e Sociedade, Curitiba, v. 11, n. 23, 2015

ISSN (versão online): 1984-3526

ISSN (versão impressa): 1809-0044

derrota de foi na primeira partida, que se tornou o primeiro jogo em que um computador venceu um campeão do mundo sob regras normais de tempo. $\mathrm{Na}$ base de dados do computador havia mais de 700 mil partidas de Mestres e Grandes Mestres (NEWBORN, 2002). Qual ser humano teria 200 milhões de posições na memória para escolher a melhor em cada jogada? Uma partida dura tipicamente 50 lances para cada jogador. No meio de jogo, em média, há 35 movimentos existentes para cada lado. Se um computador examinar dois milhões de posições por segundo, seriam necessários mais de $5,3 \times 10^{109}$ anos para esgotar todas as possibilidades de lances. Após o primeiro lance (saída das Brancas e resposta das Negras), há 400 posições diferentes. Após os quatro primeiros lances, este número se eleva a cerca de 319 bilhões. Após 10 lances o número de posições diferentes é de 169 octilhões.

Um mestre do xadrez "visualiza" em média sete jogadas à frente. $O$ norueguês Magnus Carlsen é capaz de calcular 20 jogadas com antecedência. Muitas delas são tão ousadas que os especialistas não entendem onde ele quer chegar, até que o lance se completa e todos o consideram genial. Magnus tentou explicar o inexplicável: "Muitas vezes um movimento simplesmente parece o melhor." (HARELL, 2010)

Como transformar essa habilidade em programa de computador? Como raciocinar nessas previsões ser ter que armazenar e examinar milhões de possibilidades na memória, e decidir em um tempo muito limitado (alguns minutos, pois o jogo é "com relógio")?

Um dos desafios que tenho proposto em sala de aula é resolver longe de calculadoras e computadores, a expressão $(1111)^{2}$ - número decimal, não binário. Peço para estimarem o tempo gasto na solução. Em seguida, proponho, nas mesmas condições, (1111111)2. Novamente, estimativa do tempo. Por quê é menor para a maioria dos participantes? Qual o algoritmo ou raciocínio usado nos dois casos? Finalmente, pergunto: por quê nem todos fizeram este exercício? E se tivéssemos uma máquina inteligente que, logo após uma ordem ou pedido humano, respondesse: "agora não estou com vontade de fazer" ou "repita, eu não estava prestando atenção, estava divagando" ou "não gosto de você, vou atendê-lo por último"? Vontade, atenção, afetividade são objetos de estudo da Inteligência Artificial? E a questão da "consciência artificial"?

Conclui-se que, com todos os esforços para se definir "inteligência", "inteligência artificial", "programas e sistemas inteligentes", não se conhece a maioria 
Revista Tecnologia e Sociedade, Curitiba, v. 11, n. 23, 2015

ISSN (versão online): 1984-3526

ISSN (versão impressa): 1809-0044

dos processos mentais que chamamos de raciocínio. O "paradigma conexionista" da IA estuda as chamadas "redes neurais artificiais" e se houver um computador capaz de simular o processamento e as conexões entre milhares ou milhões de neurônios artificiais, é possível reproduzir a complexidade do pensamento inteligente. Em nossos cérebros, cada neurônio forma entre mil e dez mil conexões em média. $O$ cérebro humano possui cerca de 100 bilhões de neurônios, e o número de sinapses ultrapassa 100 trilhões (ANDERSON; HINTON, 1989). Ainda não há computador capaz de simular isso. A tarefa ficou muito mais difícil, porque o cérebro humano é muito mais complicado do que se imaginava. Spencer Smith e seus colegas descobriram que os dendritos dos neurônios também fazem suas próprias "computações". Até agora, sabia-se que apenas os axônios seriam ativos, e todos os processos cerebrais seriam resultantes da atuação de conjuntos de neurônios disparando sinais de forma coordenada. Os dendritos seriam apenas a "fiação" que interliga os neurônios. "O que se pensava que fosse uma simples fiação na verdade são transistores que computam informação" (SMITH et al, 2013).

\section{INTELIGENCIA ARTIFICIAL FORTE E FRACA}

Na vertente "Inteligência Artificial Forte" será possível criar uma máquina que incorpora os conceitos de "consciência" e "ética" em uma só entidade cognitivamente equivalente a seres humanos. $\mathrm{Na}$ "Inteligência Artificial Fraca", o objetivo é "programar uma máquina para considerá-la inteligente comparada a um ser humano". Ao fim, considera impossível a construção de máquinas totalmente inteligentes. (MCCARTHY et al, 2006). Especula se as máquinas podem apresentar comportamento inteligente, enquanto que a Inteligência Artificial Forte considera a existência de mentes reais em máquinas, não simuladas. Concorda-se em geral que estados mentais são associados a estados cerebrais, devido aos progressos na tomografia cerebral. Mas, o mecanismo relacionado à consciência ainda é misterioso.

Exemplos de sistemas inteligentes podem ser encontrados em Russell e Norvig (2013), Coppin (2010), Rezende (2003) e Campos e Saito (2004), dentre os textos mais acessíveis. Estudos em Inteligência Artificial Forte podem ser vistos nos trabalhos de Preston e Bishop (2002), Bishop (2009) e Rey (2003).

Recentemente, uma carta aberta foi divulgada alertando sobre os frutos da 
Revista Tecnologia e Sociedade, Curitiba, v. 11, n. 23, 2015

ISSN (versão online): 1984-3526

ISSN (versão impressa): 1809-0044

Inteligência Artificial. Da mesma forma que ela pode trazer inúmeros benefícios, muitos sem precedentes, à humanidade, há o alerta de que a perda de controle sobre os sistemas superinteligentes resulte na escravização e até destruição da raça humana. Esta carta pode ser assinada em http://futureoflife.org/misc/open letter.

\section{PONTO DE SINGULARIDADE}

A velocidade dos computadores cresce de forma exponencial. Para o inventor Ray Kurzweil (2005), um computador de mil dólares tem hoje a mesma inteligência de um inseto. No futuro, ele se igualará à capacidade de um rato, de um homem e, finalmente, de toda a humanidade.

Hoje os significados de "inteligência artificial" e "sistemas inteligentes" estão ainda bastante desconexos quando se conversa por aí. O primeiro termo conota-se melhor por "robôs humanóides", "andróides", "organismos artificiais", "exterminadores" e o segundo com "aparelhos", "utensílios", gadgets, sistemas e produtos, sendo que, neste último "esconde-se" a automação mais elaborada, porém cujas regras foram determinadas pelo algoritmo idealizado por alguém que projetou suas funções.

Destarte, os impactos da inserção dos sistemas complexos já estão sendo sentidos. Da mesma forma que a Revolução Industrial marcou o início da substituição de tarefas "braçais" humanas por automação "simples", hoje os Sistemas Especialistas estão reunindo as conquistas dos métodos de Inteligência Artificial com a automação mais complexa, possibilitando a substituição do homem por decisões de maior nível, de maneira mais rápida e com menor chance de erros. A carta aberta supracitada fornece vários exemplos em distintas áreas de como a IA deve permanecer robusta e benéfica, e principalmente, alinhada com os interesses humanos.

Com a evolução tecnológica, há uma tendência de "deixarmos" os equipamentos (cada vez mais sofisticados) "decidirem" ou "fazerem" cada vez mais nossas tarefas. Estamos ficando mais e mais dependentes das máquinas, ou pior, da "realidade" ou "visão do mundo" que elas nos oferecem (note o que acontece quando muitos são obrigados a ficar sem conexão por muito tempo, ou ainda os relacionamentos "virtuais"). Perderemos a criatividade, pois nosso modo de vida já estará todo arranjado? Perderemos a noção do que é "real" ou "virtual"? A quem a 
Revista Tecnologia e Sociedade, Curitiba, v. 11, n. 23, 2015

ISSN (versão online): 1984-3526

ISSN (versão impressa): 1809-0044

inteligência pertencerá? Onde residirá?

\section{REFERÊNCIAS}

AXISA, F. et al. Flexible technologies and smart clothing for citizen medicine, home healthcare, and disease prevention. IEEE Transactions on Information Technology in Biomedicine, v. 9, p. 325-336, 2005.

BISHOP, M. Why Computers Can't Feel Pain. Minds and Machines, v. 19, n. 4, p. 507-516, 2009.

BOSCOV, I. Um terrível mundo novo, Veja, n. 1762 - 31 de julho de 2002. Disponível em http://veja.abril.com.br/310702/p_106.html. Acesso em 22/09/14.

BOZCHALUI, M. C.; CHENRUI, J.; SHARMA, R. Rolling Stochastic Optimization based operation of distribution systems with PVs and Energy Storages - Innovative Smart Grid Technologies Conference (ISGT) 2014. Anais...IEEE, fev. 2014. Disponível em: http://ieeexplore.ieee.org/articleDetails.jsp?arnumber=6816497. Acesso em: 8 out. 2014

CAMPBELL, M.; HOANE, A. J.; HSU, F. Deep Blue. Artificial Intelligence, v. 134, n. 1-2, p. 57-83, jan. 2002.

CAMPOS, M. M. DE; SAITO, K. Sistemas Inteligentes em Controle e Automação de Processos. [s.l: s.n.]. 2004.

$\mathrm{CAO}$, J. et al. Implications: Societal Collective Outcomes, Including Manufacturing, Convergence of Knowledge, Technology and Society: Science Policy Reports Roco, Mihail C.; Bainbridge, William S. ; Tonn, Bruce ; Whitesides, George (ed) pp.255-285 Springer International Publishing 2013 http://dx.doi.org/10.1007/978-3-319-02204-8_7

CAPDA. Subgrupo de Trabalho 2 do CAPDA - Comitê das Atividades de Pesquisa e Desenvolvimento na Amazônia. Microeletrônica e Microsistemas. 2004. Disponível em: http://www.ctpim.org.br/microeletronica.pdf. Acesso em: 10 out. 2014.

CASTRO, E. C., CARVALHO, W. F., SANTOS, N. M. GSI - Controle Inteligente - Sistemas Inteligentes - UEM, Disponível em http://ws2.din.uem.br/ ia/intelige/controle2/ . Acesso em 22/09/14

COPPIN, B. Artificial Intelligence Illuminated. [s.I: s.n.]. 2004. 768 p.

DELAC, K.; GRGIC, M. A survey of biometric recognition methods. 46th Internation Symposium Electronics in Marine, 2004., v. 16, p. 184-193, 2004.

DICK, P. K. Minority Report Gollancz: London, 2002 ISBN 1-85798-738-1 / 0-575-07478-7)

GALL, K. et al. Thermomechanics of the shape memory effect in polymers for biomedical applications. Journal of Biomedical Materials Research - Part A, v. 73, p. 339-348, 2005.

GARDNER, H. Intelligence Reframed: Multiple Intelligences for the 21st Century. [s.I: s.n.]. 1999, 292 p.

GOTTFREDSON, L. S. Mainstream science on intelligence: An editorial with 52 signatories, history, and bibliography. Intelligence, v. 24, n. 1, p. 13-23, 30 nov. 1996.

HARREL, E. A Bold Opening for Chess Player Magnus Carlsen. Time. Jan 2010.

Disponível em: http://content.time.com/time/magazine/article/0,9171,1950939,00.html. Acesso em: 10 out. 2014.

HINTON, GEOFFREY E., ANDERSON, J. A. Parallel Models of Associative Memory. 
Revista Tecnologia e Sociedade, Curitiba, v. 11, n. 23, 2015

ISSN (versão online): 1984-3526

ISSN (versão impressa): 1809-0044

Journal of Mathematical Psychology, v. 26, n. 1, p. 78-88, 1 mar. 1989.

IIDA, O. et al. Efficacy of the S.M.A.R.T. Control vs. other stents for aortoiliac occlusive disease in contemporary clinical practice. Journal of endovascular therapy: an official journal of the International Society of Endovascular Specialists, v. 20, p. 431-9, 2013.

JAUK, E. et al. The relationship between intelligence and creativity: New support for the threshold hypothesis by means of empirical breakpoint detection. Intelligence, v. 41, n. 4, p. 212-221, jul. 2013.

KURZWEIL, R. The Singularity Is Near: When Humans Transcend Biology. Viking Penguin 2011 p. 652. ISBN 0-670-03384-7

LAMPERT, C. Smart switchable glazing for solar energy and daylight control. Solar Energy Materials and Solar Cells, v. 52, n. 3-4, p. 207-221, 30 abr. 1998.

MCCARTHY, J. et al. A Proposal for the Dartmouth Summer Research Project on Artificial Intelligence. Al Magazine, v. 27, p. 12-14, 2006.

MESE, J. C. et al. Smart traffic signal system US Patents, no US6989766, 2006. Disponível em: <http://www.google.com/patents/US6989766>

MINSKY, M. Steps toward Artificial Intelligence. Proceedings of the IRE, v. 49, n. 1, 1961.

NEWBORN, M., Deep Blue: An Artificial Intelligence Milestone. [s.I.] Springer Science \& Business Media, 2003. p. 346

NOVAK, M., 50 Years of the Jetsons: Why The Show Still Matters, Smithsonian Magazine, September 19, 2012, http://www.smithsonianmag.com/history/50-years-of-the-jetsons-whythe-show-still-matters-43459669/?no-ist. Acesso em 18/09/14.

OGATA, K. Engenharia de Controle Moderno, 5a Ed., Pearson, 2011. ISBN 9788576058106.

PEREIRA, I. S. Eu, robô e a inteligência artificial forte: o homem entre a mente e a máquina. Ciências \& Cognição, v. 09, p. 150-157, 2006.

PRESTON, J. M., BISHOP, J. M. (eds.) Views Into the Chinese Room: New Essays on Searle and Artificial Intelligence Oxford University Press (2002) ISBN(s) 0199252777 9780199252770

RASHID, R. A. et al Simple Scheduling Scheme for Smart Home and Ambient Assisted Living - The Second International Conference on Informatics Engineering \& Information Science (ICIEIS2013). Anais The Society of Digital Information and Wireless Communication, 2013 Disponível em: http://sdiwc.net/digital-library/simple-schedulingscheme-for-smart-home-and-ambient-assisted-living . Acesso em: 8 out. 2014

REY, G. Searle's misunderstandings of functionalism and strong Al. View into the Chinese Room: New essays on Searle and artificial intelligence, n. 301, p. 201-225, 2003.

REZENDE, S. O. Sistemas inteligentes: fundamentos e aplicações. Manole, 2003.

RUSSELL, S.; NORVIG, P. Artificial Intelligence: A Modern Approach, Third edition. [s.I.] Campus, 2013.

SMITH, S. L. et al. Dendritic spikes enhance stimulus selectivity in cortical neurons in vivo. Nature, v. 503, n. 7474, p. 115-20, 7 nov. 2013.

SNIHIROVA, D. et al. pH-sensitive polymeric particles with increased inhibitor-loading capacity as smart additives for corrosion protective coatings for AA2024. Electrochimica Acta, v. 145, p. 123-131, nov. 2014.

STOREY, L. et al. Minority report. Nursing management (Harrow, London, England: 1994), v. 8, p. 6-9, 2002. 
Revista Tecnologia e Sociedade, Curitiba, v. 11, n. 23, 2015

ISSN (versão online): 1984-3526

ISSN (versão impressa): 1809-0044

ULRICH, I. R.; MONDADA, F.; NICOUD, J.-D. Autonomous Vaccum Cleaner. Robotics and Autonomous Systems, v. 19, p. 233-245, 1997b.

WASKAN, J. Views into the Chinese Room: New Essays on Searle and Artificial Intelligence Philosophical Review, p.277-282, 2005. 